\title{
Characterization of Leishmania Ulcers Microbiota Using Next Generation Sequencing
}

\section{RESEARCH}

\author{
Suheir Ereqat ${ }^{1, *}$, Amer Al-Jawabreh ${ }^{2,3}$, Ziad Abdeen ${ }^{4}$, Hanan Al-Jwabreh², and Abedelmajeed Nasereddin ${ }^{4}$ \\ ${ }^{1}$ Biochemistry and Molecular Biology Department-Faculty of Medicine, Al-Quds University, Abu Deis, Palestine \\ 'Leishmanises Research Unit, Jericho, Palestine, \\ ${ }^{3}$ Arab American University, Jenin, Palestine \\ ${ }^{4} \mathrm{Al}-\mathrm{Quds}$ Nutrition and Health Research Institute, Faculty of Medicine, Al-Quds University, Palestine
}

\section{A B S T R A C T}

The human skin microbiome is a major source of bacteria in cutaneous leishmaniasis (CL) ulcers following the fall of the crust and the subsequent formation of a shallow depression in the epidermis and dermis of the skin. As a result, secondary bacterial infections are frequently observed which impair the healing process. Our study aimed to investigate the bacterial communities in CL lesions using next-generation sequencing. A total of 298 patients (178 males and 120 females; the median age of 17) presenting ulcerated skin lesions suspected with CL were included in this study. CL was confirmed in 153 (51\%) cases by ITS1-PCR and/ or microscopy. Based on bacterial 16S rRNA-PCR, 92 samples were positive for the presence of bacteria, while 206 samples were negative and excluded from the microbiome study. A total of 925 Operational Taxonomic Units (OTUs) were identified and assigned to 215 genera. Despite an insignificant difference in the microbiome composition between $\mathrm{CL}$ and non-CL lesions, the phylum level analysis revealed that Actinobacteria was significantly higher in CL ulcers while Proteobacteria was significantly higher in non-CL ulcers $\left(X^{2}, P=0.039\right)$. The relative abundance of the most commonly encountered skin pathogens i. e E. coli, Pseudomonas aeruginosa, Enterobacter, Enterococcus and Acinetobacter species were significantly higher in non-CL ulcers $\left(X^{2}, P<0.05\right)$ compared to Staphylococcus aureus and Proteus mirabilis which was higher in CL ulcers $(P<0.05)$. Our data showed that bacterial communities did not cluster according to the Leishmania infection. Nonetheless, bacterial diversity was lower in CL compared to non-CL lesions. Presence of pathogenic bacteria in CL lesions such as S. aureus might exacerbate lesions, hinder diagnosis, and delay healing.
\end{abstract}

Keywords: Leishmaniasis, CL lesion, Microbiome, Next-generation sequencing.

\section{Introduction}

Cutaneous leishmaniasis (CL) is the most common form of leishmaniasis, occurring either as zoonotic or anthroponotic infections caused by different species of the genus Leishmania. In the Middle East and North Africa, zoonontic $\mathrm{CL}$ is mostly caused by Leishmania major while anthroponotic $\mathrm{CL}$ is caused by $\mathrm{L}$. tropica (Tabbabi, 2019). It is transmitted following a bite from an infected female sandfly, Phlebotomus spp. After an incubation period of several weeks to several months,

\footnotetext{
* Correspondence:

Biochemistry \& Molecular Biology Department, Faculty of Medicine, Al-Quds University, Abu Deis, Palestine.

(c) copy rights 2021: All materials in this article are protected permission requests should be addressed to Al-Quds University. www. alquds.edu
}

$\mathrm{CL}$ manifests itself starting from small erythematous papules through nodules to ulcerative lesions which later develop crusts that fall after a period time leaving a crater-like depression(Ashford, 2000; Klaus and Frankenburg, 1999; Yanik, Gurel et al., 2004). The healing or chronicity of the CL ulcer is essentially dependent on skin integrity and immune system. Shortly after sandfly inoculation, infective promastigotes evade host innate immunity to survive. The ongoing battle between the host immune response and the invading parasites will decide the fate of the disease. It is well known that $T$ cells play an essential role in the resolution of infection. Thus, individuals with a $T$ cell response, characterized 
by delayed-type hypersensitivity (DTH) and high levels of interferon $\lambda$ (IFN- $\lambda$ ), control the parasite load in their lesion compared to those with only a humoral response (Al-Jawabreh et al., 2017; Scott and Novais, 2016). However, environmental exposure and poor hygiene at the lesion site may promote polymicrobial infections (Salgado, Queiroz et al., 2016). Moreover, Leishmania parasites normally disrupt the natural skin barrier to establish cutaneous lesions that predispose to bacterial infections and can further cause dysbiosis by changing the composition of skin microbiota (Gimblet, Meisel et al., 2017; Silva-Almeida, Pereira et al., 2012). Secondary bacterial infections are frequently observed in $22 \%$ to $68 \%$ of the $\mathrm{CL}$ patients (Fontes, Carvalho et al., 2005; Vera, Macedo et al., 2006). skin microbiota was limited to culture-dependent assays with several culture-based studies reporting Staphylococcus spp, Streptococcus spp, Enterococcus spp, Pseudomonas spp, Escherichia coli, Proteus spp, Klebsiella spp and other opportunistic bacteria in CL lesions (Vera, Macedo et al., 2006; Ziaei, Sadeghian et al., 2008). The secondary bacterial infection increases tissue destruction, prolongs the duration of $\mathrm{CL}$ lesion, exacerbate lesions, hinders diagnosis, decreases the efficacy of CL treatment, and raises the probability of scar formation (Sadeghian, Ziaei et al., 2011). The skin microbiome containing a huge abundance of microorganisms which is a major source of secondary infection with several factors being reported as having the ability to alter the composition of skin microbial community such as behavioral factors, hygiene practices and use of cosmetics (Casadevall and Pirofski, 2015; Qian et al., 2012; Shin, Pei et al., 2015). Recently, new molecular approaches revealed that only about 1$2 \%$ of the skin-colonizing bacteria could be cultivated under usual conditions indicating the insufficient sensitivity of culture-based methods (Bertesteanu et al., 2014). The $16 \mathrm{~S}$ small subunit ribosomal (rRNA) gene is universal among prokaryotes and contains 9 hypervariable regions (V1-V9) of varying conservation. More conservative regions are helpful for determining the higher ranking taxa whereas more quickly evolving ones can be used for molecular identification of the majority of bacterial genera and species (Bukin et al., 2019). Based on this, microorganisms colonizing the skin fall into four different phyla: Actinobacteria, Firmicutes, Bacteroidetes and Proteobacteria (Weyrich et al., 2015).
This study aimed to reveal the bacterial microbiome composition underlying $\mathrm{CL}$ ulcers and to investigate the relative abundance of different genera and species.

\section{Methods}

\section{Subjects and samples collection}

A total of 298 patients presenting ulcerated skin lesions clinically suspected with $\mathrm{CL}$ were recruited in this study. The dermal tissue scrapings were sampled from the border of one lesion, after extensive cleaning with $70 \%$ alcohol (3 times) as previously described (Al-Jawabreh, Schnur et al., 2004). The sampling involved the outer surface layer (epidermis) and the deeper layer (dermis). All tissue scrapings were blotted on sterile filter papers for ITS1-PCRs and on slides for microscopy. The smear samples were prepared and stained with Giemsa's stain and then examined under the microscope (X100) (100x)for the presence of Leishmania amastigote forms. Patients were confirmed to be $\mathrm{CL}$ after positive results in any of the two tests, ITS1PCR or microscopic examination.

\section{DNA Extraction}

Genomic DNA was extracted from each dried tissue spots on filter papers according to manufacturers' instructions (Purelink $^{\circledR}$, Invitrogen ${ }^{\text {TM }}$, CA, USA). DNA samples were frozen at $-80^{\circ} \mathrm{C}$ until use. All samples ( $\mathrm{CL}$ and non-CL, $\mathrm{n}=298$ ) were processed with the same DNA extraction method to minimize variation in DNA yield which may affects the relative abundance of the bacteria measured at genus or species level (Kennedy, Walker et al., 2014).

\section{PCR-RFLP of the Internal Transcribed Spacer 1 (ITS1)}

All samples were amplified targeting a fragment of $300 \mathrm{bp}$ of the ITS1 gene using $400 \mathrm{nM}$ of LITSR and L5.8S primers (Schonian, Nasereddin et al., 2003). The reaction was carried out with PCR-Ready Supreme mix (Syntezza Bioscience, Jerusalem) for a total reaction volume of $25 \mu \mathrm{l}$. Amplification conditions were as described previously, in brief PCR amplification was done as followed: initial denaturation at $95^{\circ} \mathrm{C}$ for 2 min 
followed by 34 cycles consisting of denaturation at $95^{\circ} \mathrm{C}$ for $20 \mathrm{sec}$, annealing at $53^{\circ} \mathrm{C}$, and extension at $72^{\circ} \mathrm{C}$ for $1 \mathrm{~min}$. This was followed by a final extension cycle at $72^{\circ} \mathrm{C}$ for $6 \mathrm{~min}$ (el Tai, Osman et al., 2000; Schonian, Nasereddin et al., 2003). The obtained PCR products were digested with Hae III enzyme, according to the manufacturer's instructions and visualized by UV light on $2 \%$ agarose gels by electrophoresis at $120 \mathrm{~V}$ in $1 \mathrm{X}$ Trisacetate-EDTA buffer (0.04M Tris acetate and $1 \mathrm{mM}$ EDTA, pH 8).The 100 bp GeneRuler DNA ladder Mix (Fermentas, MBI) was used as the DNA molecular marker. Reference strains of L. tropica (MHOM/ AZ/1974/SAF-K27), L. major (MHOM/TM/1973/5ASKH), and L. infantum (MHOM/TN/1980/IPT1) were used as positive controls.

\section{S rRNA library preparation and sequencing}

The hypervariable $\mathrm{V} 3-\mathrm{V} 4$ region of the $16 \mathrm{~S}$ rRNA gene from each sample was amplified as previously described using the degenerate primer set (forward primer: 5'TCGTCGGCAGCGTCAGATGTG TATAAGAGACAGCCTACGGGNGGCWGCAG, reverse pr imer:5'GTCTCGTGGGCTCGGAGATGTGTATAAGAGACA GGACTACHVGGGTATCTAATCC)(Caporaso, Lauber et al., 2012). Amplification reactions were performed using X2 KAPA HiFi HotStart Ready Mix (Kappa Biosystems) with a final volume of $25 \mu \mathrm{l}$. The PCR conditions were as follows: $95^{\circ} \mathrm{C}$ for 3 minutes, followed by 25 cycles of $95^{\circ} \mathrm{C}$ for 30 seconds, $55^{\circ} \mathrm{C}$ for 30 seconds, $72^{\circ} \mathrm{C}$ for 30 seconds, then $72^{\circ} \mathrm{C}$ for 5 minutes and final hold at $4^{\circ} \mathrm{C}$. Negative controls containing nuclease-free water were used in each PCR run. Five microliters $(5 \mu l)$ of the amplified product were loaded on $2 \%$ agarose gel to visualize a band of 500bp and to confirm successful amplification. PCR products were purified using AMPure XP beads (X0.8) followed by a second round of amplification using the Nextera XT Index Kit (Illumina). The prepared libraries were normalized and then pooled at $10 \mathrm{nM}$ concentrations, $5 \mu \mathrm{l}$ of $4 \mathrm{nM}$ was denatured, and mixed with Phix control (15\%) and finally loaded onto an Illumina MiSeq machine using Miseq 500 cycle kit (Illumina). The reaction preparations and PCR conditions were described in the online 16S Metagenomic Sequencing Library Preparation guide (Illumina). The 16 rRNA gene raw sequence data were quality-filtered and analyzed using CLC Genomics Workbench 9.0 (Qiagen, Denmark).

\section{Bioinformatics and Statistical analysis}

Pairwise distances were calculated between all DNA sequence reads. Then, reads were clustered into operational taxonomic units (OTUS) at the 0.03 level, meaning that sequences that displayed $>97 \%$ similarity with each other were considered the same OTU. The taxonomic affiliation of each OTU was based on the Ribosomal Database Project (RDP-II) database. The OTUs were arranged in a data matrix where each row was a single sample and each column a specific OTU; each data point in the matrix represented the abundance of the particular OTU in the particular sample, relativized to the sampling effort, i.e. the number of reads obtained from that sample(McMurdie and Holmes, 2014). All OTUs identified to species or genus level were included in the study, while those not reaching this level were filtered out. Relative abundance of bacteria at genus/species level was displayed in the form of heat map clustered and scaled by correlation distance and average linkage. The software Rstudio, clustVis (http://www.rstudio.com/) and XLSTAT were used for data analysis and representation.

\section{Ethics}

Since this study involved the analysis of samples obtained during routine diagnostic work, patients were not asked to give their informed consent. However, patient data were anonymized for all laboratory and data analyses. The study design was approved by the ethics committee of Al-Quds Public Health Society under permission number 184/2014.

\section{Results}

\section{Study Subjects}

A total of 298 patients (178 males and 120 females; the median age was 17; and mean age \pm SD (standard deviation) was 20.98 \pm 1.04 ) presenting ulcerated skin lesions suspected with $\mathrm{CL}$ were recruited from 11 districts in Palestine from 2004 to 2016. Palestine is in the north-eastern hemisphere encompassed between the points $31^{\circ}$ $19^{\prime} 23.47^{\prime \prime} \mathrm{N}-34^{\circ} 13^{\prime} 08.78^{\prime \prime} \mathrm{E}$ in the west and $31^{\circ}$ 
$45^{\prime} 37.35^{\prime \prime} \mathrm{N}-35^{\circ} 33^{\prime} 31.43^{\prime \prime} \mathrm{E}$ in the east as well as between the points $33^{\circ} 17^{\prime} 26.79^{\prime \prime}$ N-35 $35^{\circ}$ ' 05.91' $E$ in the north and $29^{\circ} 29^{\prime} 27.51^{\prime \prime} \mathrm{N}-34^{\circ} 54^{\prime} 13.54^{\prime \prime}$ E in the south. The absolute minimum temperature was $-2 C^{\circ}$ recorded in January in the mountainous areas while the absolute maximum temperature was 48.2 $\mathrm{C}^{\circ}$ recorded in August in the Jordan valley (Jericho). The number of collected samples per district was shown in Figure 1. On examination, 192 (64.4\%) patients showed one lesion while 106 (35.6\%) partients presented multiple lesions (ranged from 2 to 12) at different body sites. The duration of the lesions ranged from 1 week to 3 months.

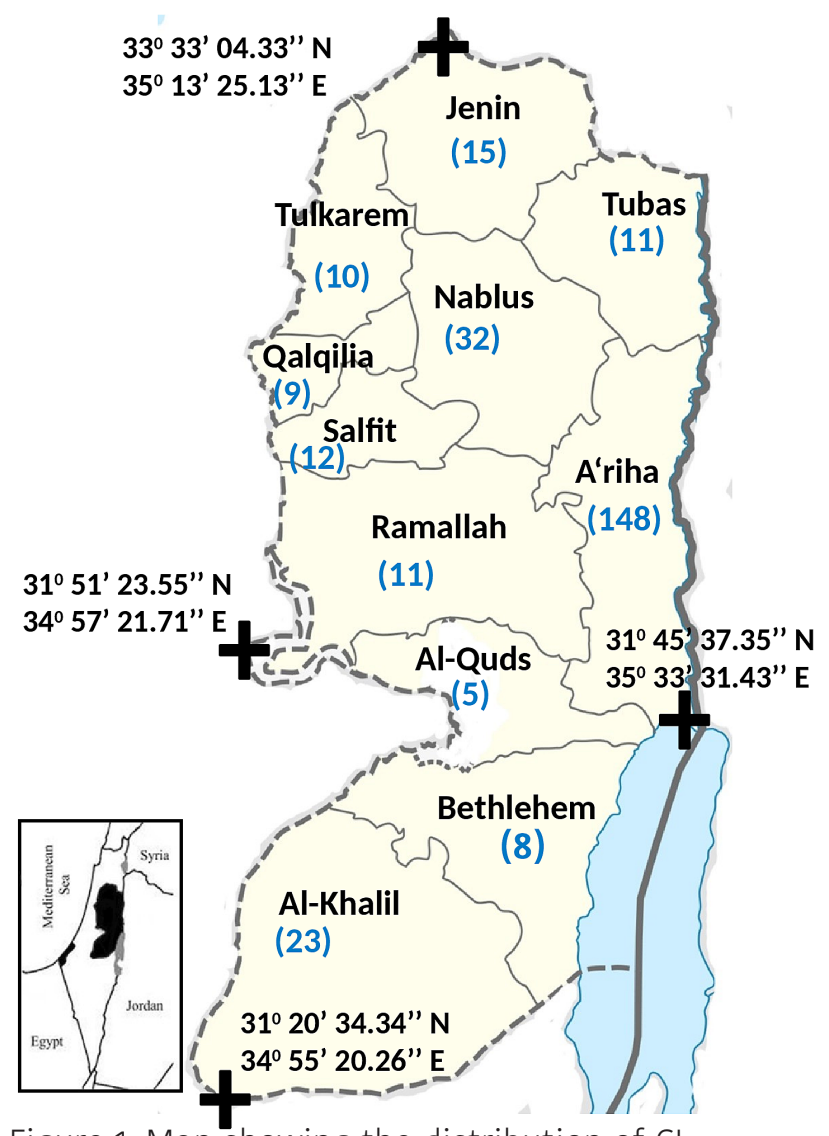

Figure 1. Map showing the distribution of $\mathrm{CL}$

suspected cases in the study area

Detection and identification of Leishmania
parasites

All samples ( $n=298$ ) were tested by ITS1- PCR and microscopic examination of Giemsa-stained smears. CL was diagnosed by demonstrating the amastigote stage of the Leishmania parasite or detecting DNA in tissue specimens spotted on filter papers. Out of the total samples, 153 (51\%) of the cases were confirmed Leishmania positive at least by one method. On the other hand, 145 (49\%) samples were negative by both tests and designated as non-CL lesions since the causative pathogen was unidentified. Of the ITS1-PCR positive samples, RFLP analysis showed $20.8 \%$ as L. major and $71.8 \%$ as L. tropica (Figure 2).

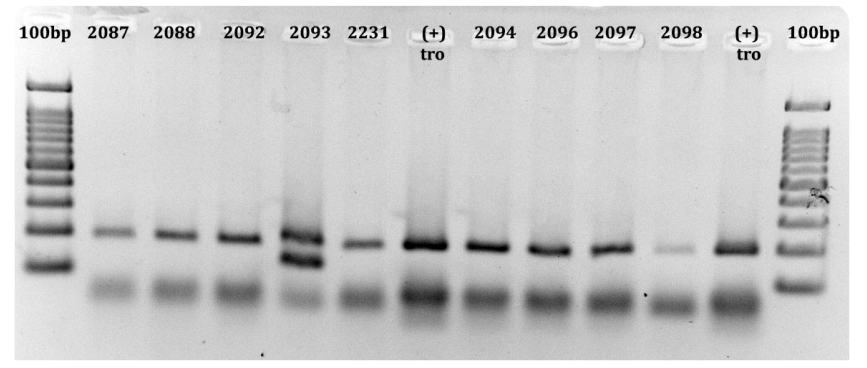

Figure 2: RFLP (Restriction fragment length polymorphism) pattern of ITS1 amplicon with Hae III. The patterns shown represent L. major (lane 5, Rec 2093) and L. tropica (rest of lanes) isolated from different patients in Palestine anonymized by record (Rec) numbers.

\section{Analysis of $16 \mathrm{~S}$ rRNA sequences}

The hypervariable $V 3$ and $V 4$ regions of the bacterial 16S rRNA gene from all DNA samples ( $C L$ and non-CL, $n=298$ ) were amplified. Results showed that $28.7 \%(44 / 153)$ of CL lesions and $33.1 \%$ (48/145) of non-CL lesions samples were positive by16S rRNA-PCR. In total, 92 samples which showed bands indicating a positive result for $16 \mathrm{~S}$ rRNA PCR were sequenced, while 206 samples were negative and, therefore, excluded from the microbiome study. Out of 92 samples, 27 (29\%) were taken from patients who received antibiotics before time of sampling, 52 samples (56.5\%) were from untreated patients and 13 (14\%) were uncertain. After the quality filter checks, 2,218,116 high quality reads were obtained: 811,295 reads were from CL ulcers and 1,406,821 reads from non-CL (Table 1$)$. The average length of the sequences was $442 \mathrm{bp}$. The OTUs were filtered down from a total of 1448 to 925, based on the genus level of identification; 896 OTUs in CL and 907 in non-CL ulcers (Table 1). All OTUs were assigned to 215 genera; 183 genera in $\mathrm{CL}$ and 197 in a non-CL group.

The 925 OTUs were, then, filtered down to 274 according to the likelihood of pathogenicity. 
Table 1. Sequence analysis result profile

\begin{tabular}{lccc}
\hline Parameter & $\begin{array}{c}\mathrm{CL} \\
\text { lesion }\end{array}$ & $\begin{array}{c}\text { Non-CL } \\
\text { lesion }\end{array}$ & Total \\
\hline No. of samples & 153 & 149 & 298 \\
$\begin{array}{l}\text { No.16S rRNA-PCR } \\
\text { positive }\end{array}$ & 44 & 48 & 92 \\
$\begin{array}{l}\text { No.of treated cases } \\
\text { prior to sampling }\end{array}$ & 33 & 46 & 76 \\
No. of reads & 811,295 & $1,406,821$ & $2,218,116$ \\
Number of genera & 183 & 197 & 215 \\
No. of OTUs & 896 & 907 & 925 \\
\hline
\end{tabular}

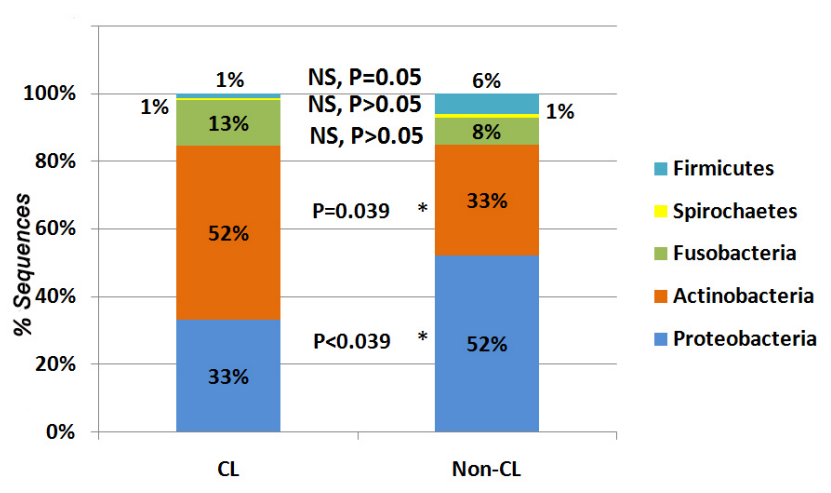

Figure 3. Diversity of potentially-pathogenic bacteria in CL ulcers vs non-CL ulcers. Colors of the stacked bargraph represent the bacterial taxa at the phyluml evel in the 274 OTUs. NS: not significant.
The 274 OTUs were collapsed into their basic 79 species and genera. Using $R$ studio software, the grand mean (mean of means) for the number of reads of CL patients was 16.8, while it was 35 for non-CL, showing a high difference in reads between the two groups $\left(P=0.013, X^{2}\right)$. Most sequences detected in both groups were assigned to five core phyla: Proteobacteria, Actinobacteria, Firmicutes, Fusobacteria, and spirochaetes with different proportions (Figure 3). The Gram-negative Proteobacteria and the Gram-positive Actinobacteria, well-known skin microbiota (Cosseau, Romano-Bertrand et al., 2016), represent the main bacteria in both types of ulcers. Cumulative percentage of sequences from the bacterial phyla Actinobacteria were significantly higher in CL ulcers ( $P=0.039)$, while Protoeobacteria was significantly higher in non-CL ulcers $(P=0.039)$. The 79 species/genera were further collapsed into 32 literature-reported skin and soft tissue pathogens in humans with varying degrees of pathogenicity, as some species were merged into their original genera (Chiller, Selkin et al., 2001; Ki and Rotstein, 2008; Moet, Jones et al., 2007). According to the bacterial pathogenicity, these genera/species were classified into two groups; most likely to cause disease and less likely, the

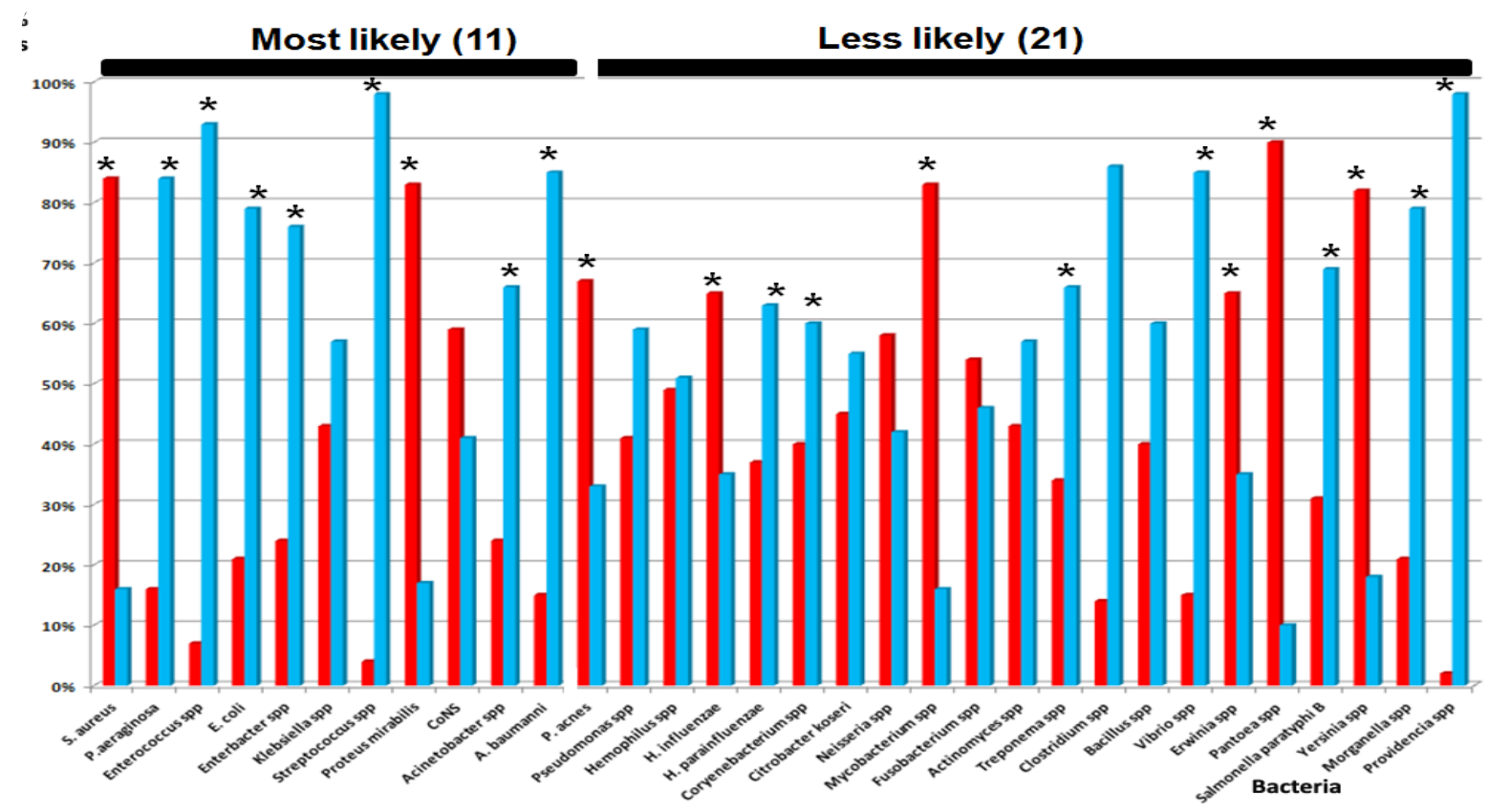

Figure 4: Relative abundances of bacterial genera/species $(n=32)$ detected in $C L$ (red bars) and non- $C L$ ulcers (blue bars). Asterisks designate statistical significance $(P<0.05)$. Klebsiella spp included $K$. pneumoniae, Acinetobacter spp included all species except A. baumannii, Pseudomonas spp included all species except $P$. aeruginosa, Propionibacterim spp included P. avidum (See supplementary files S1). 
former is known to be pathogenic and not skin flora, while the latter is usually skin flora which can become pathogenic due to reasons like weakened immune system and mode of entry like bite (Chiller, Selkin et al., 2001; Moet, Jones et al., 2007). The relative abundances of the two groups were compared in $\mathrm{CL}$ and non-CL ulcers as shown in Figure 4. In the first group, the relative abundance of $E$ coli (79\%), Pseudomonas aeruginosa (84\%), Enterobacter (76\%), Enterococcus (93\%) and Acinetobacter species (66\%) were significantly higher in non-CL ulcers $(P<0.05)$, while Staphylococcus aureus and Proteus mirabilis were higher in $\mathrm{CL}$ ulcers which represent (84\%) and (83\%) of reads, respectively $(P<0.05)$. CoNS (Coagulase-negative Staphylococci) and Klebsiella spp. were comparable in $\mathrm{CL}$ and non-CL $(P>0.05)$. The variation in the relative abundances of OTUs in $\mathrm{CL}$ and non-CL microbiome as a function of Leishmania species, prior treatment, age and gender are illustrated in the heat map (Figure 5). The bacterial genera/ species across the patients' samples clustered intermediately together on three occasions (2-6 on intensity color scale shown in Figure. 5). In the first cluster, Corynebacterium spp and Pseudomonas spp grouped with samples of $\mathrm{CL}$ ulcers. The second cluster showed Treponema spp, Haemophilus spp, Neisseria spp, and Actinomyces spp grouping mainly in samples of non-CL ulcers. While in the third cluster, $P$. aeruginosa, E. coli, A. baumannii and other Acinetobacter spp clustered

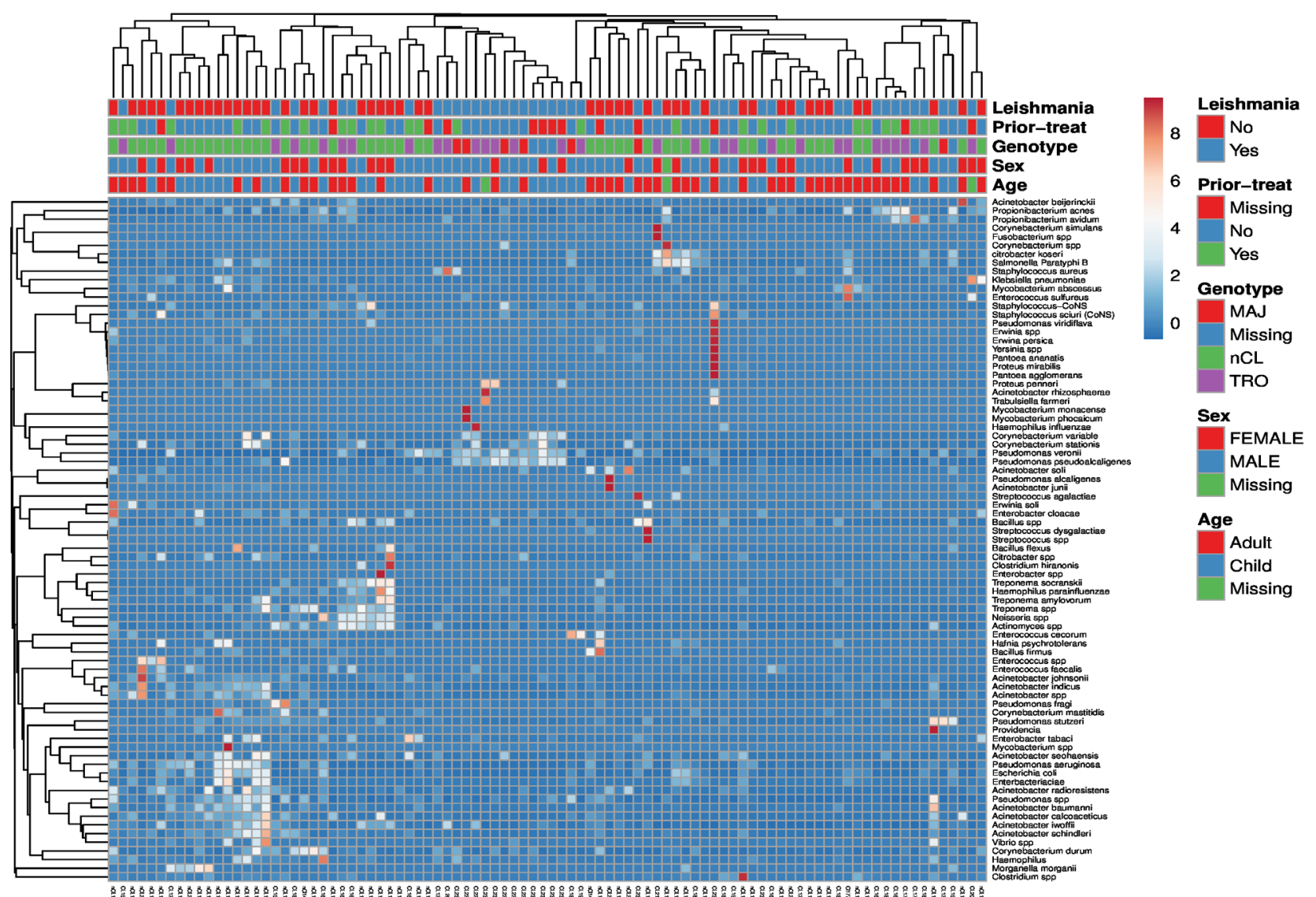

Figure 5. Heatmap of the number of reads of the bacterial genera/species (rows) and samples of patients with $\mathrm{CL}$ and non-CL ulcers (columns). Annotations on top of the heatmap show clustering of the samples based on demography and clinical picture parameters. Dendrograms are shown on the top and left side. The heat map has been color-scaled (79 rows) and clustered (both 92 columns and 79 rows). The legend of abundances (top right) is colored from low abundance (blue) to high abundance (red). 
in non-CL ulcers. One sample (CL2311) had a strong relative abundance of Enterobacteriaceae families such as Pantoea spp, Proteus spp, Erwinia spp and Yersinia spp (Figure. 5). No significant correlation was found between treatment before sampling and those without prior-treatment (Figure. 5).

\section{Discussion}

It is well-known that secondary bacterial infections can prolong the disease duration, increase tissue necrosis and result in the formation of scar (Layegh, Ghazvini et al., 2015). A study conducted on Balb/c mice reported that $\mathrm{CL}$ lesions experimentally produced by L. major may facilitate concomitant bacterial infections that interfere with the healing process. According to the results of this work, following treatment, the rate of disappearance of bacteria inoculated into the base of the tail of uninfected Balb/c mice was much faster than that of bacteria inoculated into experimentally induced $\mathrm{CL}$ lesions. These results suggested that Leishmania parasites and their metabolites might induce local immunosuppression in the lesion and thus facilitate bacterial infections (el-On, Sneier et al., 1992; Isaac-Marquez and Lezama-Davila, 2003). Our results showed that the detection rate of bacterial DNA-using 16s rRNA-PCR- in CL lesions was 28.7\%.

In both groups (CL and non-CL ulcers), most of the sequences were assigned to five core phyla: Proteobacteria, Actinobacteria, Firmicutes, Fusobacteria and Spirochaetes; which are described as the most abundant phyla of the healthy skin microbiome(Grice, Kong et al., 2009). At the phylum level, a similar microbial community with significantly different proportions was found in the microbiome of localized lesions taken from $\mathrm{CL}$ patients in Brazil: Firmicutes (54.3\%), Actinobacteria (11.7\%), Fusobacteria (11.6\%), Proteobacteria (8.7\%), and Bacteroidetes (5.1\%) (Salgado, Queiroz et al., 2016). In this study, the most abundant OTUs in CL group were from Actinobacteria phylum (52\%) followed by Proteobacteria (33\%) which may indicate a specific microbiome for Palestinian CL patients.
This unique difference between populations is supported partly by the findings of the Human Microbiome Project-HMP (http://hmpdacc.org) that showed intra and inter-individual variation in the microbiome composition within the same nation due to genetic, environmental, dietary and other unknown reasons (Human Microbiome Project, 2012). In addition, age, site of lesion and microbial interaction play role composition of skin microbiota (Mukherjee, Mitra et al., 2016; Oh, Conlan et al., 2012). Bacterial diversity was lower in $\mathrm{CL}$ lesions compared to those of non- $\mathrm{CL}$ which was associated with opportunistic contamination by commensal bacteria. E. coli and P. aeruginosa, dominated the scene in non-CL compared to $\mathrm{CL}$ lesions. Strains of $E$. coli were frequently isolated from skin and soft tissue infections and exhibited a remarkable virulence comparable to $E$. coli strains isolated from urinary tract infections and bacteremia (Petkovsek, Elersic et al., 2009). Moreover, $P$. aeruginosa a multidrug resistant pathogen also can cause diverse infections such as burn injuries and otitis externa (Hirsch and Tam, 2010). However, microbiome studies can not always identify the actual causative agent of infection by comparing the relative abundances and prevalence of pathogens, since low abundances of known causative pathogens were documented in skin ulcers (Van Leuvenhaege, Vandelannoote et al., 2017). In fact, several studies on skin microbiota have shown that most areas of the skin surface are too dry habitat for multiplication of gramnegative bacilli (Fontes, Carvalho et al., 2005). Nonetheless, $P$. aeruginosa, has been isolated from clinically-infected lesions (Moet, Jones et al., 2007). It has been demonstrated that Gram-negative bacteria, particularly Proteobacteria, represent an important component of the skin microbiota and not environmental contaminants or microbiota from other body sites (Cosseau, Romano-Bertrand et al., 2016; Grice, Kong et al., 2008).

On the other hand, the association of known Gram-positive species belonging to Firmicutes and Actinobacteria phyla to skin lesions has been previously established (Dodson, Craig et al., 2010; Kuehnert, Kruszon-Moran et al., 2006). 
Our results revealed that $84 \%$ of $\mathrm{S}$. aureus were in $\mathrm{CL}$ ulcers, which are colonizer bacteria in one third of the normal population, however, putting them into the risk of infections. The $S$. aureus sequences were relatively low which could be due to microbial interaction with coNS such as S. epidermidis, which inhibit S. aureus in a phenomenon called colonization resistant (Buffie and Pamer, 2013). We concluded that presence of pathogenic bacteria, in CL ulcers, is strongly suggestive of a concomitant bacterial infection which is in line with culture-dependent studies from Iraq, Iran, Sudan, Latin America and Brazil that showed $S$. aureus is prevalent in CL lesions (AlSamarai and AlObaidi, 2009; Fontes, Carvalho et al., 2005; Sadeghian, Ziaei et al., 2011; Ziaie and Sadeghian, 2008). The presence of pathobiome directly affects the treatment of $\mathrm{CL}$ lesions by delay in healing, disfiguring of infection site, and scar formation, therefore, several studies indicated the need to eliminate bacterial purulent infections by antibiotic treatment before antimonial administration to $\mathrm{CL}$ patients (Edrissian, Mohammadi et al., 1990; Isaac-Marquez and Lezama-Davila, 2003; Van Der Vliet, Le Guern et al., 2006). Proteus mirabilis- 59\% in CL ulcers in this study-has also been isolated from abscesses and burns (Mistry, Scott et al., 2010). Therefore, our results suggest the need to consider bacterial infections in the treatment regimen of patients with CL. We found highly abundant 16S rRNA sequences that matched $P$. acnes, of which $67 \%$ were captured in CL ulcers (Fig 3). This bacterial species is part of the normal microbiota of the skin, which thrives mainly on sebaceous body sites such as a face that formed approximately $40 \%$ of infected sites (Mukherjee, Mitra et al., 2016; Oh, Conlan et al., 2012). This high percentage of $P$. acnes induces the growth of $S$. aureus in and around the ulcer (Wollenberg, Claesen et al., 2014).

A study conducted on mice in 2017 showed that the use of topical antibiotics change the microbial make up of skin long after application. Compared to untreated mice, those who were treated with triple antibiotic ointment (TAO) exhibited an immediate and significant decrease in bacterial diversity starting after the first day of treatment and maintained for greater than 1 week post-treatment (SanMiguel, Meisel et al., 2017). In Palestine, particularly in rural areas, it is a common practice that some patients undertake empirical remedies and receive antibiotic drugs indiscriminately. Such practice may lead to changes in skin microbiota termed dysbiosis where beneficial bacteria can become pathogenic (lebba, Totino et al., 2016). We predicted that prior usage of topical or systemic medications might influence the skin microbiome. Therefore, to investigate this possible relationship, the study population was grouped into patients who reported no use of medications and those who reported use of topical and/or oral antibiotics before sampling. Although $29 \%$ of the tested lesions were treated before sampling, we found no significant differences between the microbiome of lesions in both $\mathrm{CL}$ and non-CL groups and prior antibiotic use.

This study was restricted to samples that were collected from CL-suspected patients who were referred to the Ministry of Health clinics for laboratory diagnosis. Therefore, our study limitations included: First, no samples were taken from healthy people living in the same area from which the patient samples were collected, in order to compare the results with them as control group. Second, it was difficult to recruit $C L$ and non-CL samples with the exact body site match as $\mathrm{CL}$ is not restricted to a specific body site. However, several studies showed some inter- individual differences in the skin microbiome even when matched for body site whereas other studies described the differences in the skin microbiome using samples from patients and control subjects from unmatched body sites (Fahlen, Engstrand et al., 2012).

In conclusion, the $\mathrm{CL}$ and non- $\mathrm{CL}$ ulcers have no significant difference in their microbial communities, yet, non-CL was more scattered. Nonetheless, this retrospective study does highlight that $\mathrm{CL}$ lesions are colonized with pathogenic bacteria that might complicate the clinical picture of $\mathrm{CL}$ and subsequently cause 
difficulty in diagnosis and delay in treatment. The most abundant phyla accounted for the majority of reads in the samples were members of the universal core of skin microbiome. A prospective study is needed to investigate the effect of these specific bacteria on healing time and to monitor the microbiome changes during the ulcer-healing course.

\section{References}

Al-Jawabreh, A., Dumaidi, K., Ereqat, S., Al-Jawabreh, H., Nasereddin, A., Azmi, K., Barghuthy, F., Sawalha, S., Salah, I., Abdeen, Z., 2017. Molecular epidemiology of human cutaneous leishmaniasis in Jericho and its vicinity in Palestine from 1994 to 2015. Infect Genet Evol 50, 95-101.

Al-Jawabreh, A., Schnur, L.F., Nasereddin, A., Schwenkenbecher, J.M., Abdeen, Z., Barghuthy, F., Khanfar, H., Presber, W., Schonian, G., 2004. The recent emergence of Leishmania tropica in Jericho (A'riha) and its environs, a classical focus of $L$. major. Trop Med Int Health 9, 812-816.

AlSamarai, A.M., AlObaidi, H.S., 2009. Cutaneous leishmaniasis in Iraq. J Infect Dev Ctries 3, 123-129.

Ashford, R.W., 2000. The leishmaniases as emerging and reemerging zoonoses. Int J Parasitol 30, 1269-1281.

Bertesteanu, S., Triaridis, S., Stankovic, M., Lazar, V., Chifiriuc, M.C., Vlad, M., Grigore, R., 2014. Polymicrobial wound infections: pathophysiology and current therapeutic approaches. Int J Pharm 463, 119-126.

Buffie, C.C., Pamer, E.G., 2013. Microbiota-mediated colonization resistance against intestinal pathogens. Nat Rev Immunol 13, 790-801.

Bukin, Y.S., Galachyants, Y.P., Morozov, I.V., Bukin, S.V., Zakharenko, A.S., Zemskaya, T.I., 2019. The effect of $16 \mathrm{~S}$ rRNA region choice on bacterial community metabarcoding results. Sci Data 6, 190007.

Caporaso, J.G., Lauber, C.L., Walters, W.A., Berg-Lyons, D., Huntley, J., Fierer, N., Owens, S.M., Betley, J., Fraser, L., Bauer, M., Gormley, N., Gilbert, J.A., Smith, G., Knight, R., 2012. Ultra-high-throughput microbial community analysis on the Illumina HiSeq and MiSeq platforms. ISME J 6, 1621-1624.

Casadevall, A., Pirofski, L.A., 2015. What is a host? Incorporating the microbiota into the damage-response framework. Infect Immun 83, 2-7.

Chiller, K., Selkin, B.A., Murakawa, G.J., 2001. Skin microflora and bacterial infections of the skin. I Investig Dermatol Symp Proc 6, 170-174.

Cosseau, C., Romano-Bertrand, S., Duplan, H., Lucas, O., Ingrassia, I., Pigasse, C., Roques, C., Jumas-
Bilak, E., 2016. Proteobacteria from the human skin microbiota: Species-level diversity and hypotheses. One Health 2, 33-41.

Dodson, C.C., Craig, E.V., Cordasco, F.A., Dines, D.M., Dines, J.S., Dicarlo, E., Brause, B.D., Warren, R.F., 2010. Propionibacterium acnes infection after shoulder arthroplasty: a diagnostic challenge. J Shoulder Elbow Surg 19, 303-307.

Edrissian, G.H., Mohammadi, M., Kanani, A., Afshar, A., Hafezi, R., Ghorbani, M., Gharagozloo, A.R., 1990. Bacterial infections in suspected cutaneous leishmaniasis lesions. Bull World Health Organ 68, 473-477.

el-On, J., Sneier, R., Elias, E., 1992. Leishmania major: bacterial contamination of cutaneous lesions in experimental animals. Isr J Med Sci 28, 847-851.

el Tai, N.O., Osman, O.F., el Fari, M., Presber, W., Schonian, G., 2000. Genetic heterogeneity of ribosomal internal transcribed spacer in clinical samples of Leishmania donovani spotted on filter paper as revealed by single-strand conformation polymorphisms and sequencing. Trans R Soc Trop Med Hyg 94, 575-579.

Fahlen, A., Engstrand, L., Baker, B.S., Powles, A., Fry, L., 2012. Comparison of bacterial microbiota in skin biopsies from normal and psoriatic skin. Arch Dermatol Res 304, 15-22.

Fontes, C.O., Carvalho, M.A., Nicoli, J.R., Hamdan, J.S., Mayrink, W., Genaro, O., Carmo, L.S., Farias, L.M., 2005. Identification and antimicrobial susceptibility of micro-organisms recovered from cutaneous lesions of human American tegumentary leishmaniasis in Minas Gerais, Brazil. J Med Microbiol 54, 1071-1076.

Gimblet, C., Meisel, J.S., Loesche, M.A., Cole, S.D., Horwinski, J., Novais, F.O., Misic, A.M., Bradley, C.W., Beiting, D.P., Rankin, S.C., Carvalho, L.P., Carvalho, E.M., Scott, P., Grice, E.A., 2017. Cutaneous Leishmaniasis Induces a Transmissible Dysbiotic Skin Microbiota that Promotes Skin Inflammation. Cell Host Microbe 22, 13-24 e14.

Grice, E.A., Kong, H.H., Conlan, S., Deming, C.B., Davis, J., Young, A.C., Program, N.C.S., Bouffard, G.G., Blakesley, R.W., Murray, P.R., Green, E.D., Turner, M.L., Segre, J.A., 2009. Topographical and temporal diversity of the human skin microbiome. Science 324, 1190-1192.

Grice, E.A., Kong, H.H., Renaud, G., Young, A.C., Program, N.C.S., Bouffard, G.G., Blakesley, R.W., Wolfsberg, T.G., Turner, M.L., Segre, J.A., 2008. A diversity profile of the human skin microbiota. Genome Res 18, 1043-1050.

Hirsch, E.B., Tam, V.H., 2010. Impact of multidrug-resistant 
Pseudomonas aeruginosa infection on patient outcomes. Expert Rev Pharmacoecon Outcomes Res 10, 441-451.

Human Microbiome Project, C., 2012. Structure, function and diversity of the healthy human microbiome. Nature 486, 207-214.

lebba, V., Totino, V., Gagliardi, A., Santangelo, F., Cacciotti, F., Trancassini, M., Mancini, C., Cicerone, C., Corazziari, E., Pantanella, F., Schippa, S., 2016. Eubiosis and dysbiosis: the two sides of the microbiota. New Microbiol 39, 1-12.

Isaac-Marquez, A.P., Lezama-Davila, C.M., 2003. Detection of pathogenic bacteria in skin lesions of patients with chiclero's ulcer. Reluctant response to antimonial treatment. Mem Inst Oswaldo Cruz 98, 1093-1095.

Kennedy, N.A., Walker, A.W., Berry, S.H., Duncan, S.H., Farquarson, F.M., Louis, P., Thomson, J.M., Satsangi, J., Flint, H.J., Parkhill, J., Lees, C.W., Hold, G.L., 2014. The impact of different DNA extraction kits and laboratories upon the assessment of human gut microbiota composition by $16 \mathrm{~S}$ rRNA gene sequencing. PLoS One 9, e88982.

Ki, V., Rotstein, C., 2008. Bacterial skin and soft tissue infections in adults: A review of their epidemiology, pathogenesis, diagnosis, treatment and site of care. Can J Infect Dis Med Microbiol 19, 173-184.

Klaus, S., Frankenburg, S., 1999. Cutaneous leishmaniasis in the Middle East. Clin Dermatol 17, 137-141; discussion 105-136.

Kuehnert, M.J., Kruszon-Moran, D., Hill, H.A., McQuillan, G., McAllister, S.K., Fosheim, G., McDougal, L.K., Chaitram, J., Jensen, B., Fridkin, S.K., Killgore, G., Tenover, F.C., 2006. Prevalence of Staphylococcus aureus nasal colonization in the United States, 2001-2002. J Infect Dis 193, 172-179.

Layegh, P., Ghazvini, K., Moghiman, T., Hadian, F., Zabolinejad, N., Pezeshkpour, F., 2015. Bacterial contamination in cutaneous leishmaniasis: its effect on the lesions' healing course. Indian J Dermatol 60, 211.

McMurdie, P.J., Holmes, S., 2014. Waste not, want not: why rarefying microbiome data is inadmissible. PLOS Comput Biol 10, e1003531.

Mistry, R.D., Scott, H.F., Alpern, E.R., Zaoutis, T.E., 2010. Prevalence of Proteus mirabilis in skin abscesses of the axilla. J Pediatr 156, 850-851.

Moet, G.J., Jones, R.N., Biedenbach, D.J., Stilwell, M.G., Fritsche, T.R., 2007. Contemporary causes of skin and soft tissue infections in North America, Latin America, and Europe: report from the SENTRY Antimicrobial Surveillance Program (1998-2004). Diagn Microbiol Infect Dis 57, 7-13.

Mukherjee, S., Mitra, R., Maitra, A., Gupta, S., Kumaran, S.,
Chakrabortty, A., Majumder, P.P., 2016. Sebum and Hydration Levels in Specific Regions of Human Face Significantly Predict the Nature and Diversity of Facial Skin Microbiome. Sci Rep 6, 36062.

Oh, J., Conlan, S., Polley, E.C., Segre, J.A., Kong, H.H., 2012. Shifts in human skin and nares microbiota of healthy children and adults. Genome Med 4, 77.

Petkovsek, Z., Elersic, K., Gubina, M., Zgur-Bertok, D., Starcic Erjavec, M., 2009. Virulence potential of Escherichia coli isolates from skin and soft tissue infections. J Clin Microbiol 47, 1811-1817.

Qian, J., Hospodsky, D., Yamamoto, N., Nazaroff, W.W., Peccia, J., 2012. Size-resolved emission rates of airborne bacteria and fungi in an occupied classroom. Indoor Air 22, 339-351.

Sadeghian, G., Ziaei, H., Bidabadi, L.S., Baghbaderani, A.Z., 2011. Decreased effect of glucantime in cutaneous leishmaniasis complicated with secondary bacterial infection. Indian J Dermatol 56, 37-39.

Salgado, V.R., Queiroz, A.T., Sanabani, S.S., Oliveira, C.I., Carvalho, E.M., Costa, J.M., Barral-Netto, M., Barral, A., 2016. The microbiological signature of human cutaneous leishmaniasis lesions exhibits restricted bacterial diversity compared to healthy skin. Mem Inst Oswaldo Cruz 111, 241-251.

SanMiguel, A.J., Meisel, J.S., Horwinski, J., Zheng, Q., Grice, E.A., 2017. Topical Antimicrobial Treatments Can Elicit Shifts to Resident Skin Bacterial Communities and Reduce Colonization by Staphylococcus aureus Competitors. Antimicrob Agents Chemother 61.

Schonian, G., Nasereddin, A., Dinse, N., Schweynoch, C., Schallig, H.D., Presber, W., Jaffe, C.L., 2003. PCR diagnosis and characterization of Leishmania in local and imported clinical samples. Diagn Microbiol Infect Dis 47, 349-358.

Scott, P., Novais, F.O., 2016. Cutaneous leishmaniasis: immune responses in protection and pathogenesis. Nat Rev Immunol 16, 581-592.

Shin, H., Pei, Z., Martinez, K.A., 2nd, Rivera-Vinas, J.I., Mendez, K., Cavallin, H., Dominguez-Bello, M.G., 2015. The first microbial environment of infants born by C-section: the operating room microbes. Microbiome 3, 59.

Silva-Almeida, M., Pereira, B.A., Ribeiro-Guimaraes, M.L., Alves, C.R., 2012. Proteinases as virulence factors in Leishmania spp. infection in mammals. Parasit Vectors 5, 160.

Tabbabi, A., 2019. Review of Leishmaniasis in the Middle East and North Africa. Afr Health Sci 19, 1329-1337.

Van Der Vliet, D., Le Guern, A.S., Freitag, S., Gounod, N., Therby, A., Darie, H., Buffet, P.A., 2006. Pseudomonas aeruginosa otochondritis complicating localized cutaneous leishmaniasis: prevention of mutilation by early antibiotic therapy. Am J Trop Med Hyg 75, 
270-272.

Van Leuvenhaege, C., Vandelannoote, K., Affolabi, D., Portaels, F., Sopoh, G., de Jong, B.C., Eddyani, M., Meehan, C.J., 2017. Bacterial diversity in Buruli ulcer skin lesions: Challenges in the clinical microbiome analysis of a skin disease. PLoS One 12, e0181994.

Vera, L.A., Macedo, J.L., Ciuffo, I.A., Santos, C.G., Santos, J.B., 2006. [Antimicrobial susceptibility of aerobic bacteria isolated from leishmaniotic ulcers in Corte de Pedra, BA]. Rev Soc Bras Med Trop 39, 4750.

Weyrich, L.S., Dixit, S., Farrer, A.G., Cooper, A.J., Cooper, A.J., 2015. The skin microbiome: Associations between altered microbial communities and disease. Australas J Dermatol 56, 268-274.
Wollenberg, M.S., Claesen, J., Escapa, I.F., Aldridge, K.L., Fischbach, M.A., Lemon, K.P., 2014. Propionibacterium-produced coproporphyrin III induces Staphylococcus aureus aggregation and biofilm formation. MBio 5, e01286-01214.

Yanik, M., Gurel, M.S., Simsek, Z., Kati, M., 2004. The psychological impact of cutaneous leishmaniasis. Clin Exp Dermatol 29, 464-467.

Ziaei, H., Sadeghian, G., Hejazi, S.H., 2008. Distribution frequency of pathogenic bacteria isolated from cutaneus leishmaniasis lesions. Korean J Parasitol 46, 191-193.

Ziaie, H., Sadeghian, G., 2008. Isolation of bacteria causing secondary bacterial infection in the lesions of Cutaneous Leishmaniasis. Indian J Dermatol 53, 129-131. 